\title{
Study of an Analytical Approach using Information Field-based Fuzzy Entropy
}

\author{
Jufang HU \\ College of Mechanical and Electrical Engineering, Xinyu University, China \\ 109816271@qq.com
}

\begin{abstract}
As the large number of digital devices used in our daily life, great myriad of data will be produced and how to analyze such data brings great challenge in examining the information such as information degree/measurement. Entropy is one of possible ways to analyze the information which is fuzzy and random. In order to analyze the entropy in a more precise way, this paper presents an analytical approach which uses information field-based fuzzy entropy to define the distance of information transferring function and expected information. Using the cross information and information transferring theory, this approach extends the single information source to multi-information sets adopting the fuzzy theory. Based on this approach, it is observed that, the information field entropy not only includes the independent fuzzy entropy and Shannon entropy, but also includes the cross-part. That reveals a fuzzy variable which has two independent parts: relative independent fuzziness and randomicity as well as the combanability. Additionally, when the fuzziness disappears, the information field-based entropy will be degenerated to Shannon Entropy. While, when the randomicity is getting weak, the information fieldbased entropy will be degenerated to fuzzy entropy.
\end{abstract}

Keywords: Information Analytics, Field-based; Fuzzy; Entropy.

\section{Introduction}

As the large number of digital devices used in our daily life, great myriad of data will be produced. Peter Lyman and Hal R. Varian at UC Berkeley publish "How Much Information?" which is the first comprehensive study to quantify, in computer storage terms, the total amount of new and original information created in the world annually and stored in four physical media: paper, film, optical (CDs and DVDs), and magnetic [1]. It was reported that the world produced about 1.5 Exabytes of unique information, or about 250 megabytes for every man, woman, and child on earth [2]. As the wide use of Internetbased social networks like Facebook, Twitter, Linkedin, etc, the data produced daily thus is growing rapidly. Take a typical retailer Walmart for example, it handles more than 1 million customer transactions every hour, which are imported into databases estimated to contain more than 2.5 petabytes (2560 terabytes) of data-the equivalent of 167 times the information contained in all the books in the US Library of Congress [3, 4]. With so many data, lots of information could be obtained from them [5, 6].

At its most fundamental, information is any propagation of cause and effect within a system and it is conveyed either as the content of a message or through direct or indirect observation $[7,8]$. That which is perceived can be construed as a message in its own right, and in that sense, information is always conveyed as the content of a message [9]. However, it is difficult to examine the information in terms of the uncertainty and measurement. Information entropy was proposed by Shannon so as to present the expected value of the information contained in each message [10]. In order to analyze the information from discrete events like digital devices, most of approach focuses on converting the discrete data into continuous data streams so that a function would be used 
to present the data stream to analyze its concave and convex with many operations to get the expected value [11-13]. In the theorem, it also implies that no lossless compression scheme can shorten all messages [14]. If some messages come out shorter, at least one must come out longer due to the pigeonhole principle [15]. In practical use, this is generally not a problem, for example English documents as opposed to gibberish text, or digital photographs rather than noise. And it is unimportant if a compression algorithm makes some unlikely or uninteresting sequences larger. However, the problem can still arise even in everyday use when applying a compression algorithm to already compressed data: for example, making a ZIP file of music that is already in the FLAC audio format is unlikely to achieve much extra saving in space even in the Internet-based applications $[16,17]$.

The measurement of the probability is a certain result expected to happen. In most of the practice, the information will be sent or transferred through many different media like wired or wireless communication standards. In the context of a coin flip for example, with a 50-50 probability, the entropy is the highest value of 1 . It does not involve information gain because it does not incline towards a specific result more than the other [18]. If there is a 100-0 probability that a result will occur, the entropy is 0 . In order to present the principle of minimum cross-entropy, Kullback and Leiber proposed a cross theory to talk about the information related with other information [19]. How to calculate the entropy accurately has been studied for many decades. However, in practical case, sampling and quantization are widely used to discrete the single [20]. The entropy calculation is accepted within a certain error. The similar value is always used.

In order to analyze the entropy in a more precise way, this paper presents an analytical approach which uses information field-based fuzzy entropy to define the distance of information transferring function and expected information. The analytical expressions are presented based on the information field. Using the cross information and information transferring theory, this approach extends the single information source to multiinformation sets adopting the fuzzy theory. This paper also presents the fuzzy information entropy with cross information and transferring principle.

The rest of this paper is organized as follows. Section 2 discusses about the minimum cross-entropy principle based on the possibility entropy (Shannon theory). Section 3 presents the information field-based fuzzy entropy for analytically examining the information. Section 4 gives a discussion about the approach in terms of its relation with Shannon entropy and fuzzy entropy as well as the combination of cross-information entropy. Section 5 concludes this paper by giving the future directions.

\section{Minimum Cross-entropy Principle}

Assume that we are able to predict the random variable $X$ from experience and theory. The priori probability density (PPD) $Q=\left\{q_{1}, q_{2}, \ldots, q_{n}\right\}$ which is able to generate the priori information based on the priori probability distribution. In order to verify the expectation, a testing data $x=\left(x_{1}, x_{2}, \ldots, x_{n}\right)$ could be used for calculating the quadrature of $Q$. In order to create the distribution of $x$ $P=\left\{p_{1}, p_{2}, \ldots, p_{n}\right\}$, we can set the information so that it is close to our past experiences. Thus, the minimum cross-entropy will present the minimum entropy of the cross information. Assume that the priori distribution is $Q$, the cross-entropy is:

$$
D(P, Q)=\sum_{i=1}^{n} p_{i} \ln \frac{p_{i}}{q_{i}}
$$


If it is difficult to get $Q$, according to the Laplace non-sufficient principle, $Q$ could be expressed by an average distribution $U$. The cross-entropy will be pressed as:

$$
D(P, U)=\sum_{i=1}^{n} p_{i} \ln \frac{p_{i}}{1 / n}=\ln n-\left(-\sum_{i=1}^{n} p_{i} \ln p_{i}\right)
$$

The minimum cross-entropy $D(P, U)$ equals to the maximum Shannon entropy. Since $D$ is a convex function, the local minimum value is the global minimum value. The posterior distribution $P$ could be obtained by integrating the priori distribution $Q$ with specific constraints. Based on the continuous variable, the differential entropy could be defined as:

$$
H(\mathrm{X})=-\int f(x) \log f(x) d x
$$

However, in practical case, the sampling and quantization will be used to discrete the signals so that the accuracy is accepted within a certain range using the following expressions:

$$
\begin{gathered}
H(\mathrm{X} ; \Delta \mathrm{x})=H(x, \Delta x) \approx-\sum_{i=1}^{n} f\left(x_{i}\right) \log _{2} f\left(x_{i}\right)-\log _{2} \Delta x \\
\approx-\int_{-\infty}^{+\infty} f(x) \log _{2} f(x) d x-\log _{2} \Delta x
\end{gathered}
$$

Where $\Delta x$ is the interval length after dividing $\mathrm{X}$ into $n$. From (4), the possibility distribution function (PDF) of $\mathrm{X}$ is $f(\mathrm{X})$ which is difficult to get using the priori approach. Additionally, the determination of $\Delta x$ will influence the value of $H(\mathrm{X})$. When $\Delta x$ approaches zero, $H(\mathrm{X})$ may reach $\infty$. The determination of $\Delta x$ then should meet:

$$
\sum_{i=1}^{n} f\left(x_{i}\right) \Delta x>0.999
$$

In order to differentiate the information flow with different volume, cross-entropy could be used by replacing $\Delta x$ with $\Delta x / x$, so that

$$
H(\mathrm{X} ; \Delta \mathrm{x} / \mathrm{x}) \approx-\sum_{i=1}^{n} x_{i} f\left(x_{i}\right) \log _{2}\left[\mathrm{x}_{i} f\left(x_{i}\right)\right]-\log _{2} \Delta z \sum_{i=1}^{n} x_{i} f\left(x_{i}\right) \Delta z
$$

Where $z=\ln x$. Assume $x_{1}, x_{2}, \ldots, x_{n}$ is a set of observations from $\mathrm{X}$. The estimated entropy is:

$$
H_{v}(m, n)=\frac{1}{n} \sum_{i=1}^{n} \ln \frac{y_{i+m}-y_{i-m}}{2 m / n}
$$

Where $y_{1} \leq y_{2} \leq \ldots \leq y_{n}$ is the sequencing value of $x_{1}, x_{2}, \ldots, x_{n} . m$ is a positive integer $0<m \leq n / 2$. Based on the cross-entropy principle, (7) could be improved:

$$
H_{c}(m, n)=\frac{1}{n} \sum_{i=1}^{n} \ln \frac{y_{i+m}-y_{i-m}}{c_{i} m / n}
$$


Where

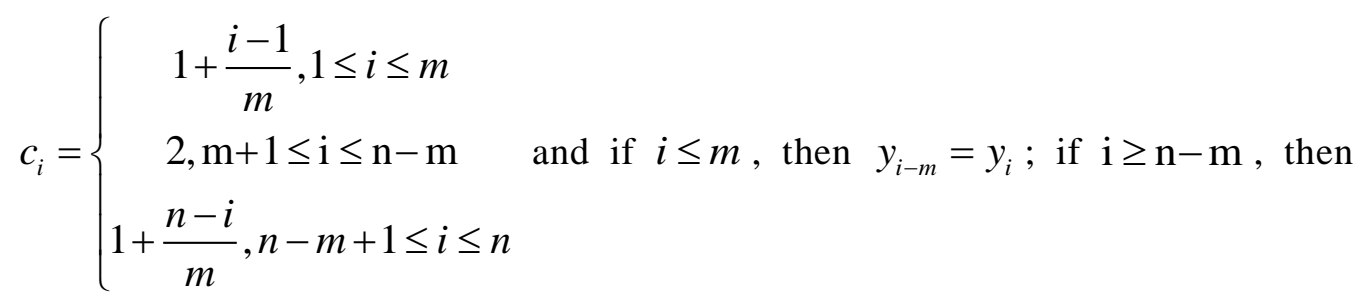$$
y_{i+n}=y_{n} .
$$

$$
H_{d}(m, n)=\frac{1}{n} \sum_{i=1}^{n} \ln \frac{z_{i+m}-z_{i-m}}{d_{i} m / n}
$$

Where

$$
\begin{aligned}
& d_{i}=\left\{\begin{array}{c}
1+\frac{i+1}{m}-\frac{i}{m^{2}}, 1 \leq i \leq m \\
2, \mathrm{~m}+1 \leq \mathrm{i} \leq \mathrm{n}-\mathrm{m}-1 \\
1+\frac{n-i}{m+1}, n-m \leq i \leq n
\end{array}\right. \\
& z_{i-m}=a+\frac{i-1}{m}\left(y_{1}-a\right)=y_{1}-\frac{m-i+1}{m}\left(y_{1}-a\right), 1 \leq i \leq m \\
& z_{i}=y_{i}, m+1 \leq i \leq n-m+1 \\
& z_{i+m}=b-\frac{n-i}{m}\left(\mathrm{~b}-y_{n}\right)=y_{n}+\frac{m+i-n}{m}\left(\mathrm{~b}-y_{n}\right), n-m \leq i \leq n
\end{aligned}
$$

\section{Information Field-based Fuzzy Entropy Analytics}

Fuzzy set cannot be determined using 0 and 1 which is very similar in some practice where typical information is uncertain. For example, $X=\{-3,-2,-1,0,1,2,3\}$, for a set $S$ which contains the integer equal and bigger than 0 , then, $S=\{0,1,2,3\}$. However, for a set $S^{\prime}$ which contains the value near 0 , it is difficult to define the elements. Thus, the membership function could be used to determine the elements of $S^{\prime}$. De Luca and Trmini defined a non-possibility entropy based on the fuzzy theory [21]. The fuzzy entropy refers to the global measurement of the uncertain statuses which are the information measurement independent with the random experiments.

The fuzzy entropy is a mapping which is defined as:

$$
\mathrm{f}: \mathrm{F}(\mathrm{X}) \rightarrow \mathrm{R}^{+}
$$

$\mathrm{F}(\mathrm{X})$ presents the whole fuzzy sub-set in the limited $\mathrm{X}$. Several characteristics are included in the fuzzy entropy analytic. First, $f(A)=0 \Leftrightarrow u_{A}(x)=\operatorname{0or} 1, \forall x \in X$, that means the fuzzy entropy of any crisp sets is 0 . Second, $\operatorname{Maxf}(A) \Leftrightarrow u_{A}(x)=1 / 2, \forall x \in X$ that indicates only one fuzzy set has the maximum fuzziness. Third, if $A \prec B$, then $f(A) \leq f(B), u_{A}(x) \leq u_{B}(x), u_{B}(x) \leq 1 / 2$, that means if $A$ is increasing, then the fuzzy entropy will be reduced. Fourth, $f(A)=f\left(A^{c}\right)$, where 
$A^{c}$ is the complement of $A$, that means the fuzzy set $A$ and its complement have the same fuzzy entropy.

Based on the characteristics, given $x_{i}(i=1,2, \ldots, n)$ with the possibility $p_{i}$, then we can get:

$$
f(A)=-\sum_{i=1}^{n} u_{A}\left(x_{i}\right) p_{i} \log p_{i}
$$

The fuzziness measurement could be presented as:

$$
f(A)=-K \sum_{x}\left[\mathrm{u}_{A}(x) \log u_{A}(x)+\left(1-u_{A}(x)\right) \log \left(1-u_{A}(x)\right)\right]
$$

Where $K$ is the normalization factor. The information distance has relations with fuzzy entropy where different fuzziness factors are related to the entropy. For example, $f(A)=d\left(A, A^{\text {near }}\right) / d\left(A, A^{\text {far }}\right)$, where $A^{\text {near }}$ and $A^{\text {far }}$ are the nearest and furthest crisp set. There are several distances such as Hamming distance, Euclidean distance, and Minkowski distance. In this paper, the information field distance is used to work out the fuzzy entropy. Assume there are two fuzzy sub-sets: $A$ and $B$

$$
\frac{S(B, A)}{S(A, B)}=\frac{\sum \operatorname{count}(A \cap B)}{\sum \operatorname{count}(B)} / \frac{\sum \operatorname{count}(A \cap B)}{\sum \operatorname{count}(A)}
$$

From (13), we can get:

$$
\frac{\sum \operatorname{count}(A)}{\sum \operatorname{count}(B)}=\frac{S(B, A)}{S(A, B)}
$$

$S(A, B)$ presents the membership of set $A$ to $B$, that is:

$$
S(A, B)=\operatorname{Degree}(A \subset B)=\frac{\sum \operatorname{count}(A \cap B)}{\sum \operatorname{count}(A)}
$$

Where, $\sum \operatorname{count}(A)=\sum_{x} u_{A}(x)$. Using the ln operation on (14), we can get:

$$
\ln \frac{\sum \operatorname{count}(A)}{\sum \operatorname{count}(B)}=\ln \frac{S(B, A)}{S(A, B)}
$$

$S(A, B)$ is the sub-set of $A$ in $B$ or the fuzzy information allocation from $B$ to $A$. $S(B, A) / S(A, B)$ is the fuzzy similarity ratio. As the decreasing of degree about $A$ and $B$, the ratio in (16) approaches 1 . Let $u_{A}(\square)$ and $u_{B}(\square)$ present the membership function, from (16), we can get:

$$
\ln \frac{\sum_{i} u_{A}\left(x_{i}\right)}{\sum_{i} u_{B}\left(x_{i}\right)}=\ln \frac{S(B, A)}{S(A, B)}
$$

For a specific $x_{i}, \mathrm{i}=1,2, \ldots, \mathrm{n}$, the required information for differentiating is:

$$
I_{1}\left(A, B: x_{i}\right)=\ln \frac{u_{A}\left(x_{i}\right)}{u_{B}\left(x_{i}\right)}
$$


The fuzzy expectation information is:

$$
I_{1}(A, B)=\sum_{i} u_{A}\left(x_{i}\right) \ln \frac{u_{A}\left(x_{i}\right)}{u_{B}\left(x_{i}\right)}
$$

In $B$, the fuzzy information to differentiate $A$ is defined as:

$I(A, B)=I_{1}(A, B)+(\bar{A}, \bar{B})=\sum_{i=1}^{n}\left[u_{A}\left(x_{i}\right) \ln \frac{u_{A}\left(x_{i}\right)}{u_{B}\left(x_{i}\right)}+\left(1-u_{A}\left(x_{i}\right)\right) \ln \frac{\left(1-u_{A}\left(x_{i}\right)\right)}{\left(1-u_{B}\left(x_{i}\right)\right)}\right]$

The information is called the asymmetry information distance from two fuzzy sets. Let $A_{F}$ presents a fuzziest set where for each $x, u_{A_{F}}=0.5$. Then the fuzzy entropy and $I\left(A, A_{F}\right)$ have the relation:

$$
f(A)=1-(1 / n \ln 2) I\left(A, A_{F}\right)
$$

For the symmetry fuzzy distance of two fuzzy set, we can get:

$$
\begin{aligned}
D(A, B) & =I(A, B)+I(B, A) \\
= & \sum_{i=1}^{n}\left[\left(u_{A}\left(x_{i}\right)-u_{B}\left(x_{i}\right)\right) \ln \frac{u_{A}\left(x_{i}\right)}{u_{B}\left(x_{i}\right)}+\left(u_{B}\left(x_{i}\right)-u_{A}\left(x_{i}\right)\right) \ln \frac{1-u_{A}\left(x_{i}\right)}{1-u_{B}\left(x_{i}\right)}\right]
\end{aligned}
$$

Thus, the fuzzy entropy could be defined as:

$$
\mathrm{H}(\mathrm{A})=\frac{D\left(A, A^{\text {near }}\right)}{D\left(A, A^{f a r}\right)}
$$

Where where $A^{\text {near }}$ and $A^{\text {far }}$ are the nearest and furthest non-fuzzy set. Since $0 \leq D\left(A, A^{\text {near }}\right) \leq D\left(A, A^{\text {far }}\right) \leq \infty$, thus, $0 \leq \mathrm{H}(\mathrm{A}) \leq 1$.

Assume there are several fuzzy subset in $X$. They are close related to each other. Let $X^{+}=\left\{x / x \in X, u_{A}(x) \geq u_{B}(x)\right\}, X^{-}=\left\{x / x \in X, u_{A}(x)<u_{B}(x)\right\}$. In order to analyze the entropy, the single entropy and union entropy could be obtained:

$$
\begin{gathered}
\left.f(A)=-K \sum_{i=1}^{n}\left(u_{A}\left(x_{i}\right) \log u_{A}\left(x_{i}\right)+\left(1-u_{A}\left(x_{i}\right)\right) \log \left(1-u_{A}\left(x_{i}\right)\right)\right)\right) \\
\left.f(B)=-K \sum_{i=1}^{n}\left(u_{B}\left(x_{i}\right) \log u_{B}\left(x_{i}\right)+\left(1-u_{B}\left(x_{i}\right)\right) \log \left(1-u_{B}\left(x_{i}\right)\right)\right)\right) \\
\begin{aligned}
& f(A \cup B)=-K \sum_{x \in X}\left(u_{A}\left(x_{i}\right) \vee u_{B}\left(x_{i}\right)\right) \log \left(u_{A}\left(x_{i}\right) \vee u_{B}\left(x_{i}\right)\right) \\
&+\left(1-u_{A}\left(x_{i}\right) \vee u_{B}\left(x_{i}\right) \log \left(1-u_{A}\left(x_{i}\right) \vee u_{B}\left(x_{i}\right)\right)\right) \\
&=-K\left[\sum_{x \in X^{+}} u_{A}\left(x_{i}\right) \log u_{A}\left(x_{i}\right)+\left(1-u_{A}\left(x_{i}\right)\right) \log \left(1-u_{A}\left(x_{i}\right)\right.\right. \\
& \sum_{x \in X^{-}} u_{B}\left(x_{i}\right) \log u_{B}\left(x_{i}\right)+\left(1-u_{B}\left(x_{i}\right)\right) \log \left(1-u_{B}\left(x_{i}\right)\right]
\end{aligned}
\end{gathered}
$$

From (24)-26), the conditional entropy could be calculated by: 


$$
\begin{aligned}
f(A / B)= & -K \sum_{x \in X^{+}}\left[\left(u_{A}(x) \log u_{A}(x)-u_{B}(x) \log u_{B}(x)+\right.\right. \\
& \left.\left(1-u_{A}(x)\right) \log \left(1-u_{A}(x)\right)-\left(1-u_{B}(x)\right) \log \left(1-u_{B}(x)\right)\right] \\
f(B / A)= & -K \sum_{x \in X^{+}}\left[\left(u_{B}(x) \log u_{B}(x)-u_{A}(x) \log u_{A}(x)+\right.\right. \\
& \left.\left(1-u_{B}(x)\right) \log \left(1-u_{B}(x)\right)-\left(1-u_{A}(x)\right) \log \left(1-u_{A}(x)\right)\right]
\end{aligned}
$$

In order to present the information field-based fuzzy entropy analytical approach, let $X=[1,100]$, which is a set. $Q$ and $Y$ indicate two fuzzy sets whose membership functions are:

$$
\begin{aligned}
& u_{Q}(x)=\left\{\begin{array}{c}
0,0 \leq x \leq 50 \\
{\left[1+\left(\frac{x-50}{5}\right)^{-2}\right]^{-1}, 50<x \leq 100}
\end{array}\right. \\
& u_{Y}(x)=\left\{\begin{array}{c}
1,0 \leq x \leq 25 \\
{\left[1+\left(\frac{x-25}{5}\right)^{2}\right]^{-1}, 25<x \leq 100}
\end{array}\right.
\end{aligned}
$$

Let $X$ is discrete then we can get $X^{\prime}=[1,2,3, \ldots, 100]$, according to the proposed approach, we can get:

$$
\begin{array}{ll}
H(Q)=11.94 & H(Y)=12.92 \\
H(Q \cup Y)=21.56 & H(Q \cap Y)=3.29 \\
H(Q / Y)=8.65 & H(Y / Q)=9.62 \\
T(Q, Y)=0.28 & T(Y, Q)=0.25
\end{array}
$$

\section{Discussions}

In most of practical applications, fuzzy entropy and Shannon entropy will be integrated. Assume there is a discrete possibility space $(X, P)$, fuzzy set $A$ is defined in $X$. The possibility of $X=x_{i}$ is $p_{i}$. Then, the information field-based entropy could be calculated by:

$$
A X=p_{i} H_{I F}(A, P)=-\sum_{i=1}^{n} p_{i}\left(u_{i} \log u_{i}+\left(1-u_{i}\right) \log \left(1-u_{i}\right)\right)
$$

The global average uncertainty is:

$$
H_{I F}^{G A U}(A, P)=H_{S}(P)+H_{I F}(A, P)
$$

Where, $H_{S}(P)$ is the Shannon entropy. Let the discrete fuzzy variable $X$ has the value of $A_{1}, A_{2}, \ldots, A_{n}, P\left\{X=A_{i}\right\}=\mathrm{p}_{i}$ and $H\left(A_{i}\right)=h_{i}$. Then, the information fieldbased entropy is:

$$
H(X)=\sum_{i=1}^{n}\left(1+h_{i}\right) p_{i} \log \left(1 / p_{i}\right)+h_{i}\left(1+p_{i} \log \left(1 / p_{i}\right)\right)
$$


If a single fuzzy event $\left\{X=A_{i}\right)$ with the Shannon entropy whose product with $p_{i}$ is $s_{i}$. Then (33) could be simplified as:

$$
H(X)=\sum_{i=1}^{n}\left(1+h_{i}\right) s_{i} \log +h_{i}\left(1+s_{i}\right)=\sum_{i=1}^{n}\left(s_{i}+h_{i}+2 s_{i} h_{i}\right)
$$

From (34), it could be observed that, the information field entropy not only includes the independent fuzzy entropy and Shannon entropy, but also includes the cross-part. That reveals a fuzzy variable which has two independent parts: relative independent fuzziness and randomicity as well as the combanability. Based on (34), several findings could be obtained. When the fuzziness disappears, the information field-based entropy will be degenerated to Shannon Entropy. While, when the randomicity is getting weak, the information field-based entropy will be degenerated to fuzzy entropy. When $A_{i}$ degenerates to a crisp set, the fuzziness of $X$ will be disappeared. Then, $H(X)=\sum_{i=1}^{n} p_{i} \log \left(1 / p_{i}\right), H(X)$ is the Shannon entropy. When the randomicity disappears, $X$ only has one value $A . H(X)=\mathrm{H}(\mathrm{A}), H(X)$ is the fuzzy entropy. When $P\left\{X=A_{i}\right\}=1 / n$ and $X=A_{i} \equiv 1 / 2(\mathrm{i}=1,2, . ., \mathrm{n}), H(X)$ has the maximum value.

Let $X$ has two value $A$ and $B$ whose membership functions are:

$$
u_{A}(x)=\left\{\begin{array}{c}
x+2,-2<x \leq-1 \\
-x,-1<x \leq 0 \\
0, \text { others }
\end{array} \quad u_{B}(x)=\left\{\begin{array}{c}
x, 0<x \leq 1 \\
2-\mathrm{x}, 1<x \leq 2 \\
0, \text { others }
\end{array}\right.\right.
$$

$$
P\{X=A\}=1 / 4 \text { and } P\{X=B\}=3 / 4 \text {, thus, we can get: }
$$

$$
H(A)=H(B)=2 \quad s_{1}=(1 / 4) \ln (1 / 4)=0.35
$$

$s_{2}=(3 / 4) \ln (3 / 4)=0.22$

According to (34), we can get: $H(X)=6.81$.

\section{Summary}

Fuzzy entropy is one of the most important parts in the information theory. This paper introduces an analytical approach to investigate the entropy using information field-based principle which considers the combination of the information. Two key characteristics relative independent fuzziness and radomicity are integrated into the analytical approach based on the single entropy, union entropy and condition entropy. This approach is able to describe the fuzzy variable with random and fuzzy uncertainty feature.

Several contributions of this paper are significant. Firstly, using the approach proposed in this paper, it is observed that the information field entropy not only includes the independent fuzzy entropy and Shannon entropy, but also contains the cross-part. That means a fuzzy variable will has two independent parts: relative independent fuzziness and randomicity as well as the combanability. Secondly, the information field-based entropy will be degenerated to Shannon Entropy when the fuzziness disappears. Thirdly, the information field-based entropy will be degenerated to fuzzy entropy when the randomicity is getting weak. 
Several future research will be carried out to enhance this research. In the first place, when the $X$ degenerates into a common single variable, what is the changing trends of $H(X)$ ? For exploring the trends, the impact degree of fuzziness and randomicity should be further studied. Secondly, if two fuzzy sets have some similarities, how to estimate the entropy if only one of the sets could be determined. Finally, practical testing with real-life data could be used to examine the feasibility and practicality of the proposed approach in the near future. Finally, as the large number of data generated from different sectors which are using digital devices widely [22], Big Data Analytics could be integrated into this research. Thus, this research could be implemented into practical cases.

\section{References}

[1] P. Lyman and H. Varian, "How much information 2003?", (2004).

[2] S. LaValle, E. Lesser, R. Shockley, M. S. Hopkins and N. Kruschwitz, "Big data, analytics and the path from insights to value", MIT Sloan Management Review, vol. 21, (2013).

[3] V. Mayer-Schönberger and K. Cukier, "Big data: A revolution that will transform how we live, work, and think", Houghton Mifflin Harcourt, (2013).

[4] R.Y. Zhong, G. Q. Huang, S. L. Lan, Q. Y. Dai, C. Xu and T. Zhang, "A Big Data Approach for Logistics Trajectory Discovery from RFID-enabled Production Data", International Journal of Production Economics, vol. 165, (2015), pp. 260-272.

[5] S. Rodríguez, J. F. De Paz, G. Villarrubia, C. Zato, J. Bajo and J. M., "Multi-Agent Information Fusion System to manage data from a WSN in a residential home", Information Fusion, vol. 23, (2015), pp. 4357.

[6] Y. Chen, B. Zhao, J. Zhang, Y. Zheng and J. Wang, "An Improved Method for Cortical Surface Identification based on Local Information", International Journal of Signal Processing, Image Processing and Pattern Recognition, vol. 6, no. 4, (2013), pp. 467-474.

[7] R. Y. Zhong, Q. Dai, T. Qu, G. Hu and G. Q. Huang, "RFID-enabled Real-time Manufacturing Execution System for Mass-customization Production", Robotics and Computer-Integrated Manufacturing, vol. 29, no. 2, (2013), pp. 283-292.

[8] D. Li, F. Yang and H. Wei, "Research on Corn Region Extraction of Remote Sensing Image Based on Fusion of PSO Parameters Optimization and Adaboost_SVM", International Journal of Signal Processing, Image Processing and Pattern Recognition, vol. 8, no. 8, (2015), pp. 361-372.

[9] Z. Shunli, "Compressed Sensing Method Application in Image Denoising", International Journal of Signal Processing, Image Processing and Pattern Recognition, vol. 8, no. 1, (2015), pp. 203-212.

[10] C. E. Shannon, "Prediction and entropy of printed English", Bell system technical journal, vol. 30, no. 1, (1951), pp. 50-64.

[11] R. Y. Zhong, G. Q. Huang, Q. Y. Dai and T. Zhang, "Estimation of Lead Time in the RFID-enabled Real-time Shopfloor Production with a Data Mining Model", Proceeding of The 19th International Conference on Industrial Engineering and Engineering Management, (2012); ChangSha, P.R China.

[12] J. Liang and Z. Shi, "The information entropy, rough entropy and knowledge granulation in rough set theory", International Journal of Uncertainty, Fuzziness and Knowledge-Based Systems, vol. 12, no. 1, (2004), pp. 37-46.

[13] R. Valencia-Torres, G.-H. Sun and S.-H. Dong, "Quantum information entropy for a hyperbolical potential function”, Physica Scripta, vol. 90, no. 3, (2015), pp. 035205.

[14] Z. Li, J. Gu, H. Zhuang, L. Kang, X. Zhao and Q. Guo, "Adaptive molecular docking method based on information entropy genetic algorithm”, Applied Soft Computing, vol. 26, (2015), pp. 299-302.

[15] T. Day, "Information entropy as a measure of genetic diversity and evolvability in colonization", Molecular ecology, vol. 24, no. 9, (2015), pp. 2073-2083.

[16] A. Yang and S. Chen, "Object recognition with image set based on kernel information entropy", 12th International Conference on Fuzzy Systems and Knowledge Discovery (FSKD), IEEE, (2015).

[17] R. Y. Zhong, S. Lan, C. Xu, Q. Dai and G. Q. Huang, "Visualization of RFID-enabled shopfloor logistics Big Data in Cloud Manufacturing", The International Journal of Advanced Manufacturing Technology, (2015), pp. 1-12.

[18] R.Y. Zhong, G. Q. Huang, Q. Y. Dai and T. Zhang, "Mining SOTs and Dispatching Rules from RFIDenabled Real-time Shopfloor Production Data", Journal of Intelligent Manufacturing, vol. 25, no. 4, (2014), pp. 825-843.

[19] A. Salama, "Separation data balancing utilizing the maximum entropy approach", International journal of mineral processing, vol. 47, no. 3, (1996), pp. 231-249.

[20] A. Elaraby, H. B. M. A. El-Owny, M. Heshmat, M. Hassaballah and A. A. Rardy, "A Novel Algorithm for Edge Detection of Noisy Medical Images", International Journal of Signal Processing, Image Processing and Pattern Recognition (IJSIP), vol. 6, no. 6, (2013), pp. 365-374. 
[21] P. Burillo and H. Bustince, "Entropy on intuitionistic fuzzy sets and on interval-valued fuzzy sets", Fuzzy sets and systems, vol. 78, no. 3, (1996), pp. 305-316.

[22] R. Y. Zhong, S. T. Newman, G. Q. Huang and S. Lan, "Big Data for supply chain management in the service and manufacturing sectors: Challenges, opportunities, and future perspectives", Computers \& Industrial Engineering, in press, (2016).

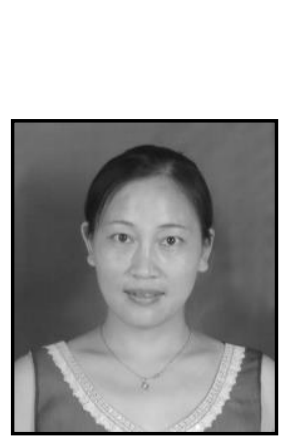

\begin{abstract}
Author
Jufang Hu, Associated Professor from Xinyu University. She graduated from Anhui University of Technology and Guangdong University of Technology with bachelor and master degrees respectively. Her research interests include embedded technology and image processing. She has published over 50 papers in international journals and conferences.
\end{abstract}

